\title{
LUXACIÓN COXOFEMORAL EN UN PONY SHETLAND: DESCRIPCIÓN DE UN
} CASO

\section{COXOFEMORAL LUXATION IN A SHETLAND PONY: CASE REPORT}

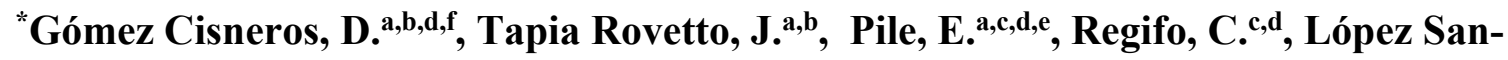

\section{Román Jf.}

${ }^{a}$ Departamento de Clínicas y Cirugías, Facultad de Medicina Veterinaria-Universidad de Panamá, ${ }^{b}$ Médico Veterinario, Complejo Hospitalario Veterinario de Corozal, Universidad de Panamá. ${ }^{\mathrm{c}}$ Enfermedades Transmisibles y Salud Pública, Facultad de Medicina VeterinariaUniversidad de Panamá. ${ }^{\mathrm{d}}$ Sistema Nacional de Investigación - SENACYT, ${ }^{\mathrm{e}}$ INDICASAT -

Adjunto de la Facultad Med. Veterinaria ${ }^{\mathrm{f}}$ Departamento de Medicina y Cirugía Animal, Facultad de Medicina Veterinaria, Universidad Complutense de Madrid, Madrid, España.

*Autor de correspondencia: david_gomez09@hotmail.com

\section{RESUMEN}

La luxación coxofemoral es una causa relativamente rara de claudicación en caballos, observándose en potros y caballos miniaturas. Como causas de luxación han sido descritos: traumatismos severos, fracturas de ilion, sobreextensión violenta y fijación de rótula. Se describe un caso, remitido al Complejo Hospitalario Veterinario de Corozal- Universidad de Panamá, de un pony Shetland, el cual presentaba una cojera grado 4/5 del miembro posterior izquierdo con atrofia de la musculatura del bíceps femoral y glúteos. Se realizó radiografía digital simple de pelvis, observándose luxación de la articulación coxofemoral. Una vez realizado el diagnóstico, se decidió realizar el tratamiento quirúrgico, que consistió en una ostectomía de la cabeza del fémur. El pronóstico es reservado en estas lesiones, pero en nuestro caso, se realizó seguimiento durante 5 meses observándose en ese tiempo menor atrofia muscular, pero manteniéndose una claudicación $2 / 5$ en la extremidad.

Palabras clave: Luxación coxofemoral, ostectomía. 


\begin{abstract}
Coxofemoral luxation is a relatively rare cause of lameness in horses, observed in foals and miniature horses. As causes of luxation, severe trauma, ilium fractures, violent overextension and patellar fixation, have been described. A case of a Shetland pony is described, referred to the Complejo Hospitalario Veterinario de Corozal- Universidad de Panamá, which had a 4/5 lameness of the left hind limb and muscle atrophy of the biceps femoris and gluteus. It was decided to perform a simple digital radiography of the pelvis, and a coxofemoral joint luxation was observed. Once made the diagnosis, it was decided to perform surgical treatment, in terms of an ostectomy and excision of the femoral head. The prognosis is reserved in these lesions, but in our case, monitoring was carried out for 5 months, where less muscle atrophy was observed, but still remained $2 / 5$ degree lameness.
\end{abstract}

Keywords: coxofemoral luxation, ostectomy

\title{
INTRODUCCIÓN
}

La luxación coxofemoral es causa relativamente rara de claudicación en caballos, observándose frecuentemente en potros y caballos miniaturas (Malark et al, 1992, GarcíaLópez et al, 2001; Tóth et al, 2007; Amitrano et al, 2014). La articulación coxofemoral en caballos posee una gran musculatura, además de fuertes ligamentos accesorios de la cabeza del fémur y un acetábulo profundo que previenen de cierta manera la luxación de esta articulación (Bennett et al, 1977; Clegg, y Butson, 1996; Hendrickson, 2002). La causa más frecuente de luxación es por trauma severo, aunque se han descrito también por fracturas de ilion, sobreextensión violenta y fijación de rótula (Bennett et al 1977; Clegg and Butson 1996; García-López 2010).

Entre los signos clínicos puede observarse grados variables de cojera (de grado 3-5/5), acortamiento de la extremidad, rotación de la extremidad, crepitación, asimetría y atrofia muscular (García Calvo et al, 2011, Amitrano et al, 2014).

Este caso describe los signos clínicos, diagnóstico y tratamiento de un pony Shetland con luxación coxofemoral. 


\section{DETALLES DEL CASO}

Se describe un caso, remitido al Complejo Hospitalario Veterinario de CorozalUniversidad de Panamá (SIADVET), de un pony Shetland, macho entero, 6 años de edad y $110 \mathrm{~kg}$ de peso (Figura 1). Los procedimientos realizados al caballo se realizaron con consentimiento informado del propietario. En la historia clínica, el veterinario informa que el propietario encontró el caballo claudicando en el prado, 2 meses antes del ingreso. Estuvo bajo tratamiento con fenilbutzona oral $(4.4 \mathrm{mg} / \mathrm{kg})$, pero como no se observó mejoría, fue referido a consulta.

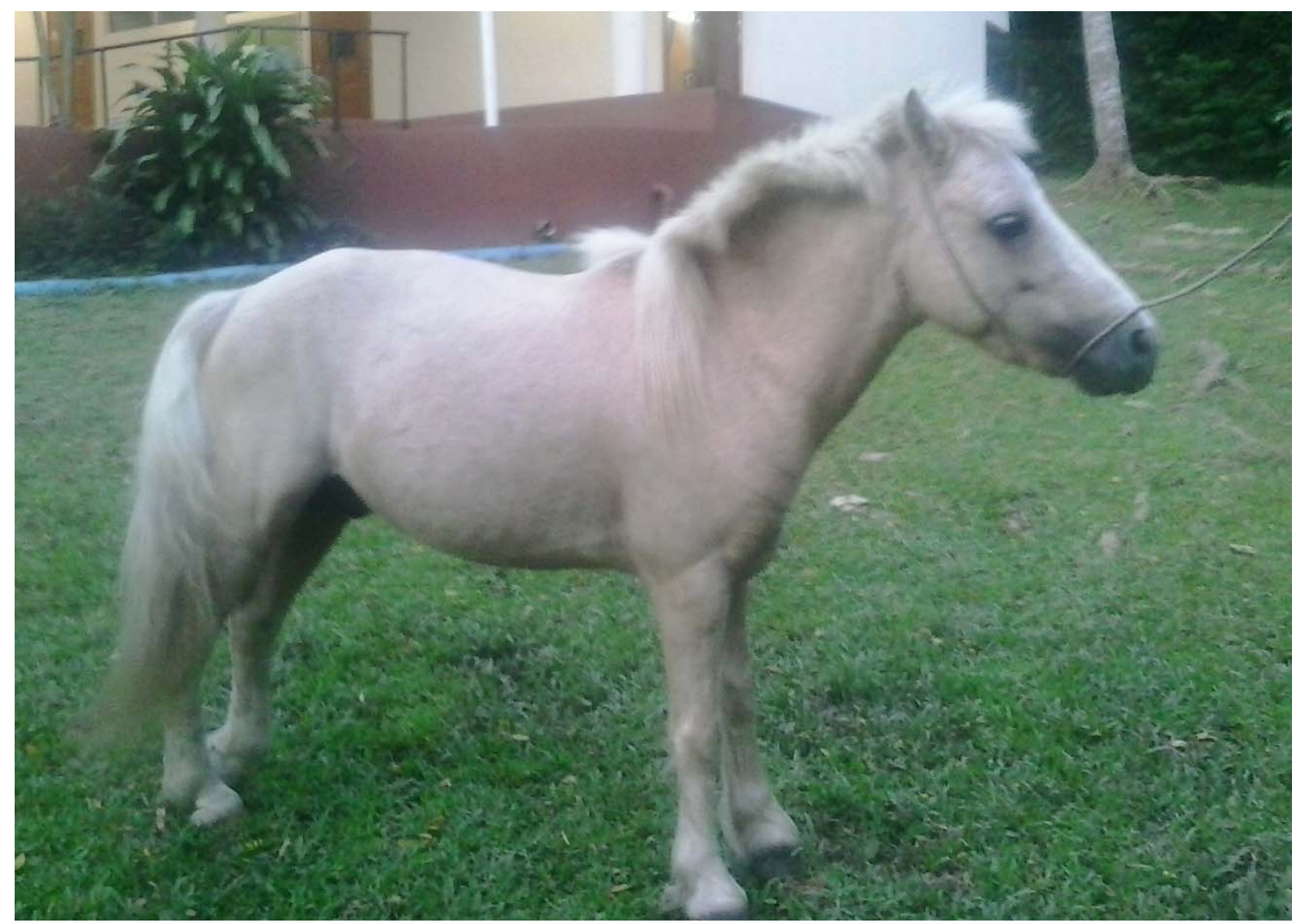

Figura 1: Pony Shetland atendido. (Imagen cortesía del Complejo Hospitalario de CorozalUniversidad de Panamá).

A la exploración clínica se observó un animal con las constantes fisiológicas dentro de la normalidad. Presentaba una cojera aguda grado 4/5 del miembro posterior izquierdo, atrofia de la musculatura del bíceps femoral y glúteos, dolor a la flexión de esa articulación, rotación hacia lateral y acortamiento del miembro afectado.

Se realizó radiología digital simple, con proyección latero-lateral y ventro-dorsal de la pelvis bajo anestesia general, además de proyecciones radiográficas de la articulación femorotibiorotuliana. En el estudio radiológico se observó luxación de la articulación. El la 
proyección latero-lateral se observó un desplazamiento hacia dorsal y craneal de la cabeza del fémur (Figura 2).

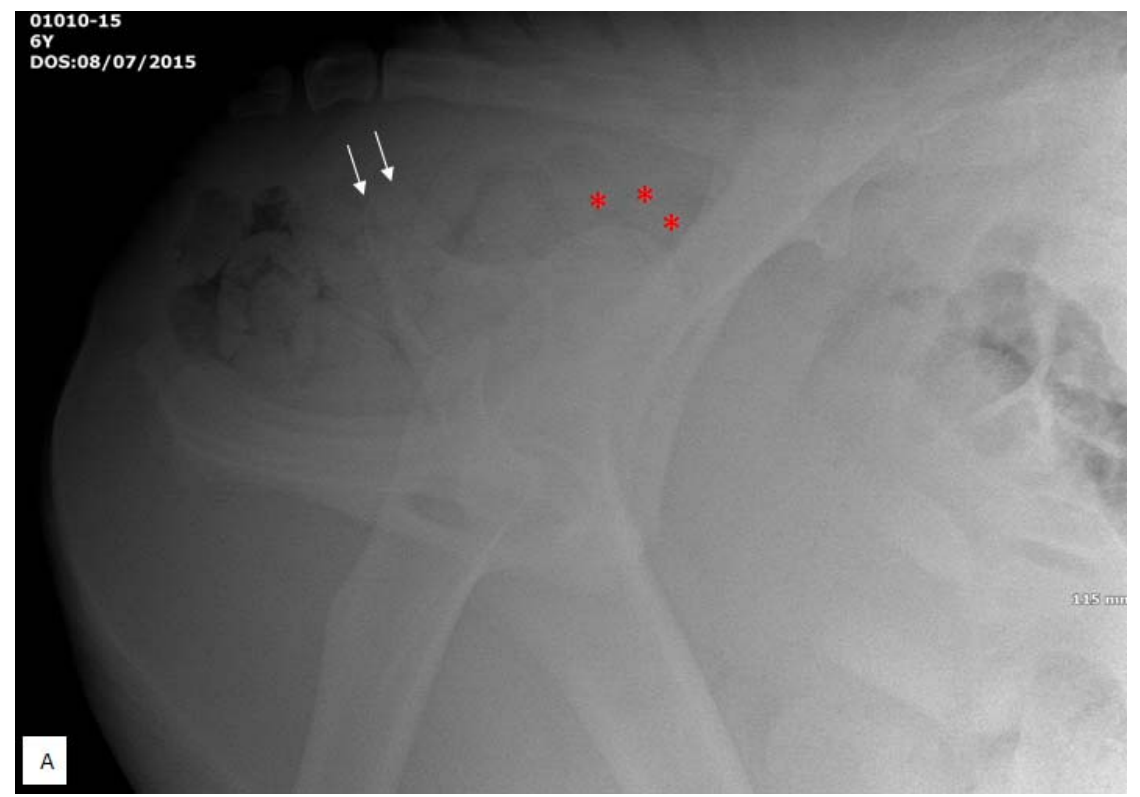

Figura 2: Proyección latero-lateral de pelvis donde se observa luxación de la articulación coxofemoral, con desplazamiento hacia dorsal y craneal de la cabeza del fémur (asteriscos) y trocánter mayor del fémur (flechas blancas). (Imagen cortesía del Complejo Hospitalario de Corozal-Universidad de Panamá).

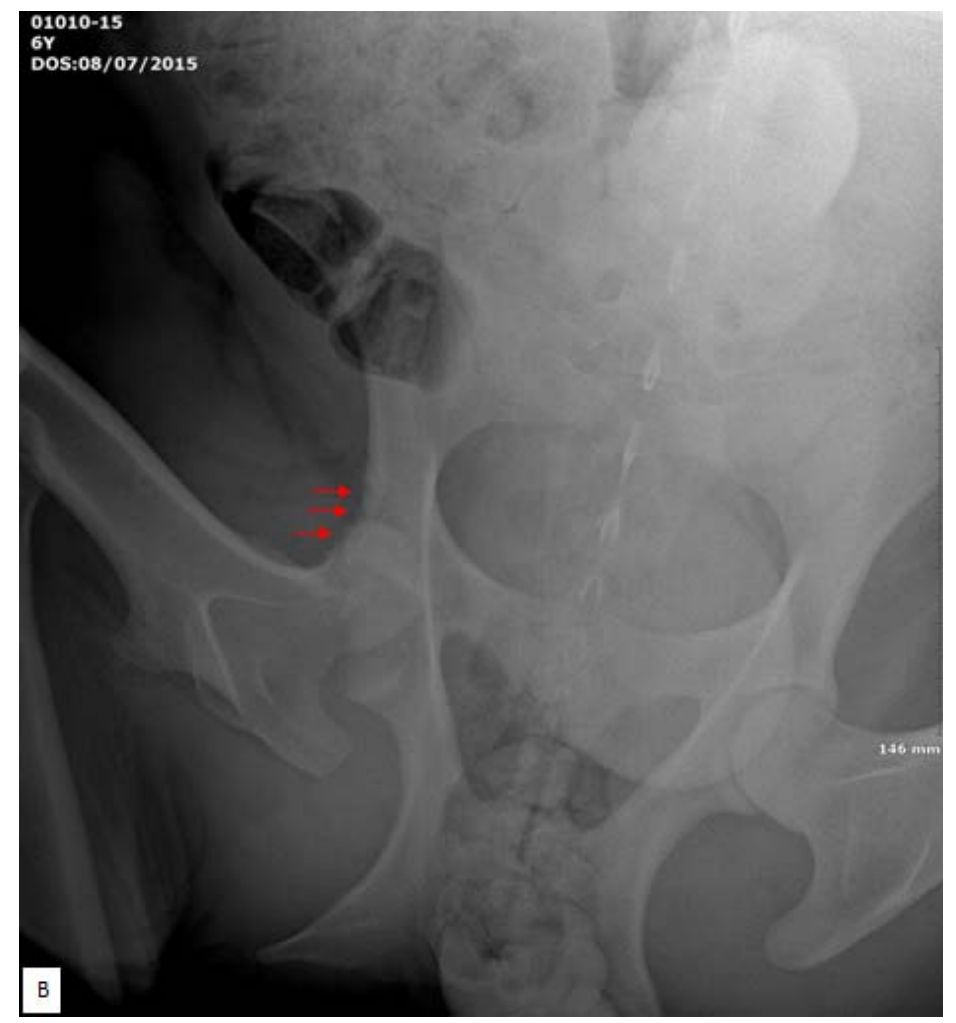

Figura 3: Proyección ventro-dorsal de pelvis, donde se observa zonas de proliferación ósea y desgaste en el acetábulo y del isquium (flechas rojas).(Imagen cortesía del Complejo Hospitalario de Corozal-Universidad de Panamá). 
En la proyección ventro-dorsal se observó luxación, además de encontrar zonas de proliferación ósea en el acetábulo y zonas de la pelvis (Figura 3). No se observaron hallazgos radiográficos relevantes en la articulación femoro-tibiorotuliana.

Una vez realizado el diagnóstico radiológico, se decidió realizar el tratamiento quirúrgico, consistente en una osteotomía de la cabeza del fémur. El pony se colocó en decúbito lateral derecho bajo anestesia general, y la se preparó quirúrgicamente la región glútea izquierda. Se realizó un abordaje cráneo-dorsal de la articulación coxofemoral, a través de una incisión curva en la piel de $25 \mathrm{~cm}$ de largo, sobre el trocánter mayor. Los músculos glúteos femoral superficial y medio, los músculos bíceps y fascia lata, fueron divididos y retraídos. Los músculos glúteos profundo y vasto lateral fueron sometidos a miotomía parcial y disección roma. La articulación fue expuesta y el material de fibrocartílago libre fue eliminado y el acetábulo desbridado. La cabeza del fémur se cortó cerca de la diáfisis del mismo con una sierra oscilante y los bordes acetabulares fueron recortados (Figura 4). Los músculos, fascia y capas subcutáneas se suturaron con material reabsorbible, y la piel con nylon. El tratamiento post-operatorio consistió en la administración de antiflamatorios no esteriodeos sistémicos (fenilbutazona $4.4 \mathrm{mg} / \mathrm{kg}$ IV) y antibióticos (ceftiufur $4 \mathrm{mg} / \mathrm{kg}$ ) durante 5 días, además de la curación diaria de la incisión.

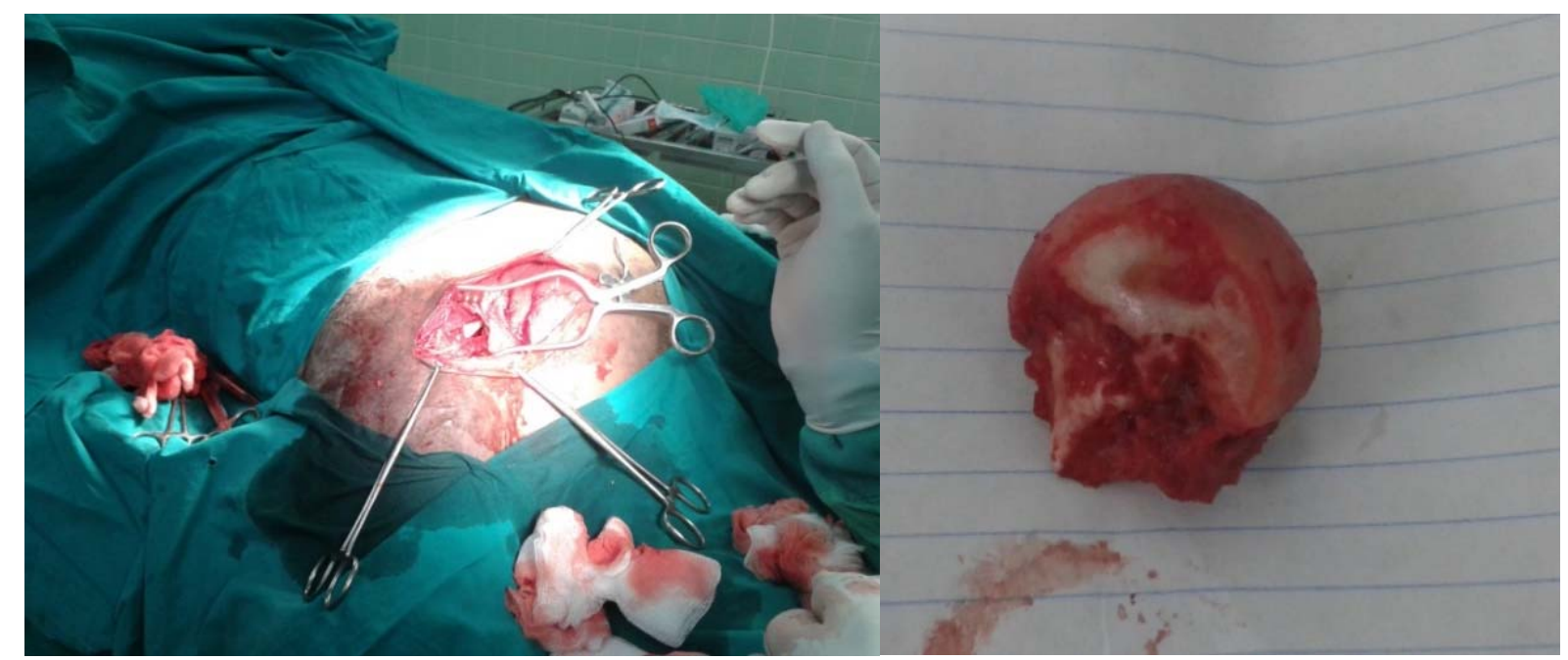

Figura 4: Abordaje quirúrgico de la articulación coxofemoral (izquierda) y cabeza del fémur (derecha). Se observa el desgaste de la cabeza del fémur provocado por la luxación. (Imagen cortesía del Complejo Hospitalario de Corozal-Universidad de Panamá). 


\section{DISCUSIÓN}

La luxación coxofemoral es una condición rara, observándose en un $0.1 \%$ de las enfermedades articulares en caballos (Barr et al, 2014), y su tratamiento representa un reto para los clínicos (García-Lopez 2010). Se ha descrito la reducción de luxaciones con vendajes (Clegg, y Butson, 1996) y técnicas quirúrgicas como la transposición del trocánter mayor del fémur, reconstrucción capsular sintética o la artroplastia (García-López et al, 2001; GarcíaLópez, 2010; Kuemmerle y Furst, 2011), cuando lleva mucho tiempo de luxado. En nuestro caso, se decidió utilizar la ostectomía de la cabeza del fémur, ya que por el tiempo transcurrido no partecía indicada la reducción por medio de vendajes de la luxación coxofemoral, observando radiográficamente desgaste del acetábulo y cabeza del fémur (García Calvo et al, 2011; Clegg y Comerford, 2007; Toth et al, 2007).

El pronóstico es reservado en estos casos (Malark et al, 1992), aunque se han descrito casos donde pueden vivir muchos años después de la ostectomía (García Calvo et al, 2011). En nuestro caso, se realizó seguimiento 5 meses después de la cirugía, observándose mayor desarrollo muscular en la extremidad izquierda, pero manteniéndose una claudicación de grado $2 / 5$.

\section{BIBLIOGRAFÍA}

Amitrano, F.N., Gutierrez-Nibeyro, S.D. and Joslyn, S.K. 2014. Radiographic diagnosis of craniodorsal coxofemoral luxation in standing equids. Equine Vet. Educ. 26, 255-258. doi: 10.1111/eve. 12156

Barr, R. S. and Fairburn, A. J. 2014. Aspects of coxofemoral luxations in the horse. Equine vet. Educ. 26 (5) 259-261. doi: 10.1111/eve.12171

Bennett, D., Campbell, J.R. and Rawlinson, J.R. 1977. Coxofemoral luxation complicated by upward fixation patella in the pony. Equine vet. J. 9, 192-194. doi: 10.1111/j.20423306.1977.tb04027.x

Clegg, P.D. and Butson, R.J. 1996. Treatment of a coxofemoral luxation secondary to upward fixation of the patella in a Shetland pony. Vet. Rec. 138, 134-137. doi: $10.2746 / 095777307 X 238525$

Clegg, P.D. and Comerford, E.J. 2007. Coxofemoral luxation - how does our knowledge of treatment in other species help us in the horse? Equine Vet. Educ. 19, 482-483. doi: $10.2746 / 095777307 X 238525$. 
García Calvo, L. A., Martín-Cuervo, M, Peña, E., Fruto, J., Jiménez, J. and Ezquerra, L. J. 2011. Femoral head excision after coxofemoral luxation in an Arab filly: Four years follow-up. Equine vet. Educ. 23 (7) 346-352. doi: 10.1111/j.2042-3292.2010.00187.x

Garcia-Lopez, J.M., Boudrieau, R.J. and Provost, P.J. 2001. Surgical repair of coxofemoral luxation in a horse. J. Am. Vet. Med. Ass. 219, 1254-1258. doi: 10.2460/javma.2001.219.1254

García-López, J.M. 2010. Coxofemoral luxations in the horse: surgical options and challenges. Equine Vet. Educ. 22, 554-556. doi: 10.1111/j.2042-3292.2010.00135.x

Hendrickson, D.A. 2002. The coxofemoral joint. In: Adams' Lameness in Horses, 5th edn., Ed: T.S. Stashak, Lippincott Williams \& Wilkins, Philadelphia. pp 1039-1042.

Kuemmerle, J. and Furst, A.E. 2011. Treatment of a coxofemoral luxation in a pony using a prosthetic capsule technique. Vet. Surg. 40, 631-635. doi: 10.1111/j.1532950x.2011.00837.x

Tóth, F., Adair, H.S., Holder, T.E.C. and Schumacher, J. 2007. Femoral head ostectomy to treat a donkey for coxofemoral luxation. Equine Vet. Educ. 19, 478-481. doi: $10.2746 / 095777307 X 220470$.

Malark JA, Nixon AJ, Haughland MA, Brown MP. 1992. Equine coxofemoral luxations: 17 cases (1975-1990). Cornell Vet. Jan;82(1):79-90. 